\title{
NEW APPROACHES FOR ARCHAEOLOGICAL FEATURE EXTRACTION OF AIRBORNE IMAGING SPECTROSCOPY DATA
}

\author{
G. Verhoeven, M. Doneus, C. Atzberger, M. Wess, M. Rus, M. Pregesbauer, C. Briese
}

\section{ARCHAEOLOGICAL AIRBORNE IMAGING SPECTROSCOPY}

Airborne hyperspectral scanning involves the mapping of a scene's wavelength intensity, accomplished by measuring upwelling electromagnetic radiation (reflected and/or emitted) in a multitude of contiguous narrow spectral bands. The end product consists of spatially co-registered two-dimensional images, each of them representing a spectral band that is typically just about ten nanometres wide. In this sense, imaging spectroscopy yields a three-dimensional data cube $(x, y, \lambda)$ in which the first two are the spatial dimensions, whereas the third axis contains a spectral dimension: a digital number (DN) that represents the sampled and quantized at-sensor radiance $\mathrm{L}$ for that particular waveband. In post-processing, reflectance (or emissive characteristics) can be calculated from these DNs. Through a combination of all spectral data acquired from a particular spatial location, every individual pixel of the final image holds the complete reflectance or emission spectrum (known as spectral signature) of the material that was sampled at that specific location. Since this spectral signature can be obtained for every pixel in the image, the technique is also called airborne imaging spectroscopy (AIS).

AIS data sets offer new possibilities for archaeological research, because traditional multi-spectral sensors spectrally undersample the true signature. Despite this, archaeological AIS has occupied only a small niche in the field of remote sensing during recent decades. Even now, its application in archaeological research is limited and most results are not entirely convincing for practical applications. Aside from the common, archaeologically insufficient ground-sampling distance of 2-3 m, the technical processing of these data typically does not go beyond the calculation of band ratios and a principal component analysis.

This means that at least two broad problems need to be solved (assuming that the image geometry can be correctly handled) before AIS data can become of real archaeological interest. The first problem relates to the huge amount of available information that is not directly accessible to the human eye, making data mining approaches necessary. The second problem relates to data quality. Indeed, as the upwelling electromagnetic radiation is recorded in small bands that are only about ten nanometres wide, the signal received by the sensor is quite low compared to sensor noise and possible atmospheric perturbations. The high spatial resolution of the AIS data sets, which is of the utmost importance when flown for archaeological purposes, requires a small IFOV (instantaneous field-of-view) further limiting the useful signal stemming from the ground. For these reasons, noise reduction techniques are necessary.

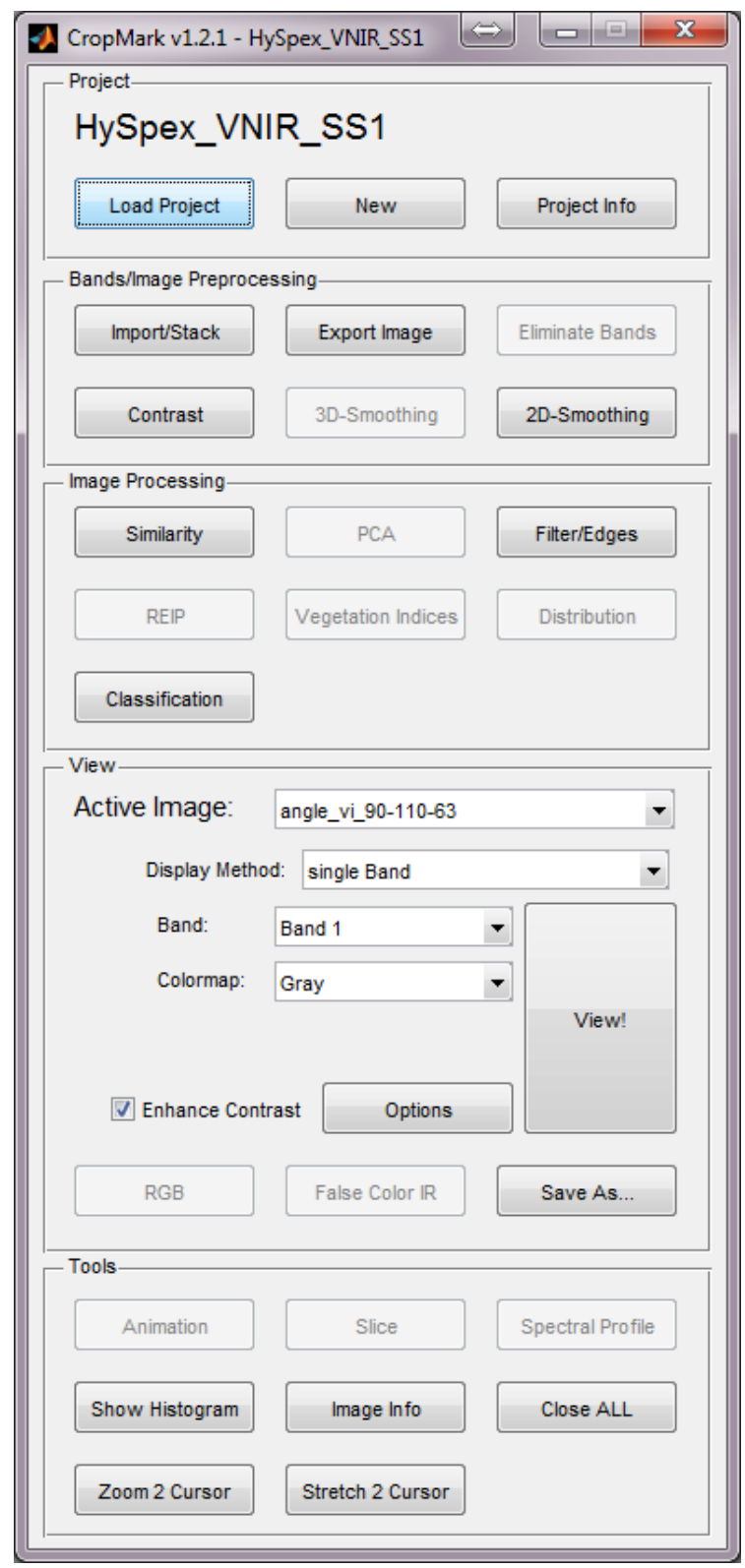

Figure 1: The GUI for the Matlab CropMark toolbox.

\section{MATLAB TOOLBOX}

To deal with these issues, a Matlab toolbox called "CropMark" and an accompanying user-friendly graphical user interface (GUI) were developed (Figure 1) to help the image analyst (not necessarily a specialist in remote sensing nor in imag- 

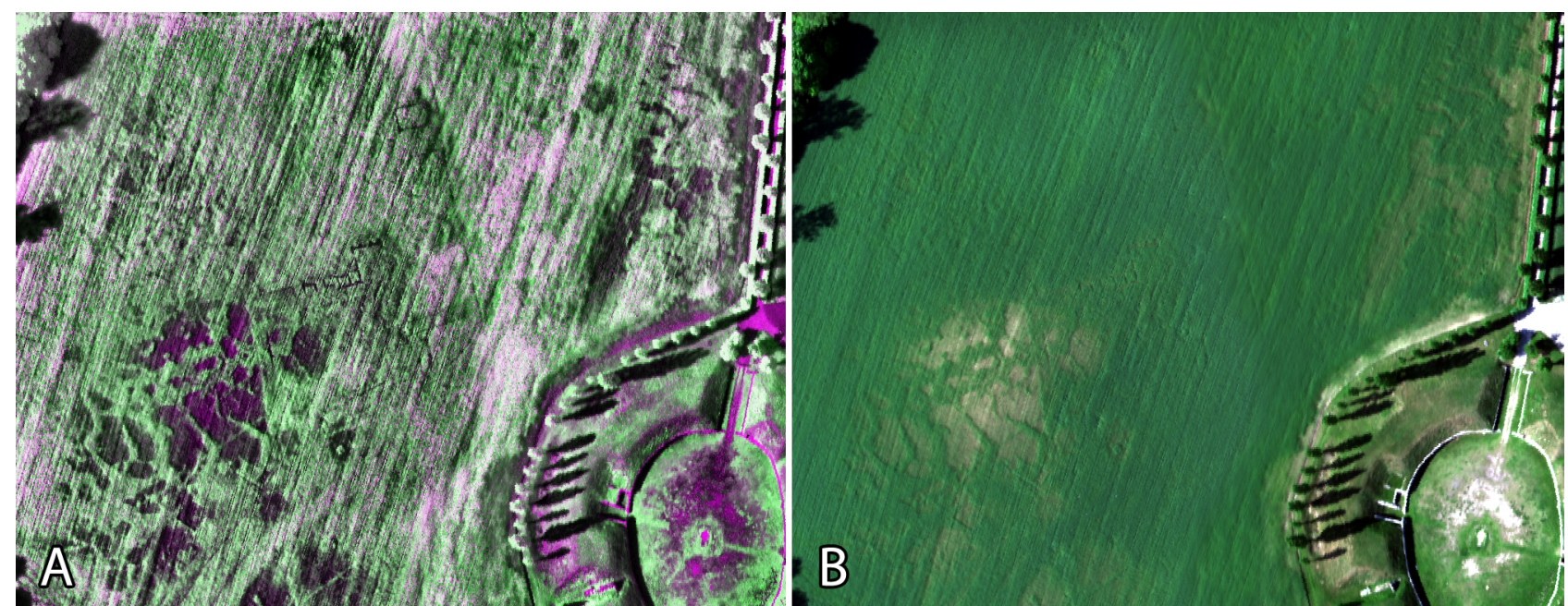

Figure 2: (A) False colour composite $(R=$ band $3, G=$ band $2, B=$ band 3$)$ created by means of the REIP algorithm; $(B)$ shows the same area in a conventional $R G B$ image. Both images are histogram-stretched.

ing spectroscopy) in getting the most information out of the recorded $3 \mathrm{D}$ data cube. As the main focus for the toolbox is archaeological AIS prospection, the aim was to visualize the data and to highlight possible crop or soil marks. Powerful, not commercially available filters based on the Whittaker smoother (Atzberger and Eilers, 2011) were implemented to deal with the noise in the hyperspectral bands. The user can visualize any sequence of individual bands in an animated way, or look at the first few principle components. Additionally, various standard and optimized hyperspectral vegetation indices were realised. Areas with a similar spectral signature can be highlighted and compared to a user-selected pixel or region of interest. The user can further test the usefulness of a large set of edge detection algorithms.

\section{AIS PROCESSING}

To extract archaeologically-relevant information from several hyperspectral datasets acquired in different seasons above the Roman town of Carnuntum (Austria), several new and notcommonly applied algorithms were tested.

The first technique is called the Red Edge Inflection Point (REIP) derived from spectrally smoothed and oversampled signatures. Previous research has already proven the archaeological usefulness of the red edge spectral band when used in a simple vegetation index (Verhoeven and Doneus, 2011). The REIP algorithm, however, will not use the complete red edge spectrum, but looks for the location of the highest gradient in the red edge spectral profile curve. Using the very powerful Whittaker smoother, the input AIS data are not only smoothed but also interpolated to a user-defined number of fictional "bands" between the original spectral bands. Afterwards, the REIP is calculated on a pixel-by-pixel basis and generates three image layers:

- Layer 1: band or wavelength of REIP location;

- Layer 2: value of the slope of the reflectance curve at the REIP position;

- Layer 3: Reflectance value at REIP.

Figure 2 shows the revealing power of a REIP false-colour composite $(\operatorname{Red}=$ band 3 , Green $=$ band 2 , Blue $=$ band 3$)$ and $a$ histogram-stretched conventional aerial image (2B) of the same scene: i.e. the Roman gladiator school (ludus) located next to the civil amphitheatre in Carnuntum (Austria). Obviously, the overall contrast of the archaeological marks is much higher in image 2A (which is also histogram-stretched), while certain features only become visible in the REIP product.

A second technique is called distribution fitting. It is known that different distributions exist (e.g. normal/Gaussian, Poisson, gamma, beta). All of these are characterised by one or more parameters. The well-known normal curve, for instance, is completely described using the mean and the standard deviation. In the distribution fitting approach, the histogram of the complete spectral signature is calculated on a pixel-basis. Afterwards, a user-defined curve is fitted to this histogram, while the values of the parameters describing this curve form the pixel values of the newly generated image bands. Using a gamma curve (described by its shape parameter $k$ and scale parameter $\theta$ ), zones with a higher biomass (indicated in black in Figure $3 \mathrm{~A}$ ) could be delineated much better compared to a conventional, histogramstretched RGB aerial image.

\section{CONCLUSION}

By programming a specific AIS Matlab toolbox, a tool was created to test currently available AIS processing techniques as well as validate the value of completely new information extraction techniques. The examples given here prove that more specifically curve fitting and the visualisation of the red edge inflection point can yield new insights into crop vigour and crop stress.

\section{ACKNOWLEDGEMENTS}

The Ludwig Boltzmann Institute for Archaeological Prospection and Virtual Archaeology (archpro.lbg.ac.at) is based on an international cooperation of the Ludwig Boltzmann Gesellschaft (A), the University of Vienna (A), the Vienna University of Technology (A), the Austrian Central Institute for Meteorology and Geodynamic (A), the office of the provincial government of Lower Austria (A), Airborne Technologies GmbH (A), RGZMRoman Germanic Central Museum Mainz (D), RAÄ -Swedish 

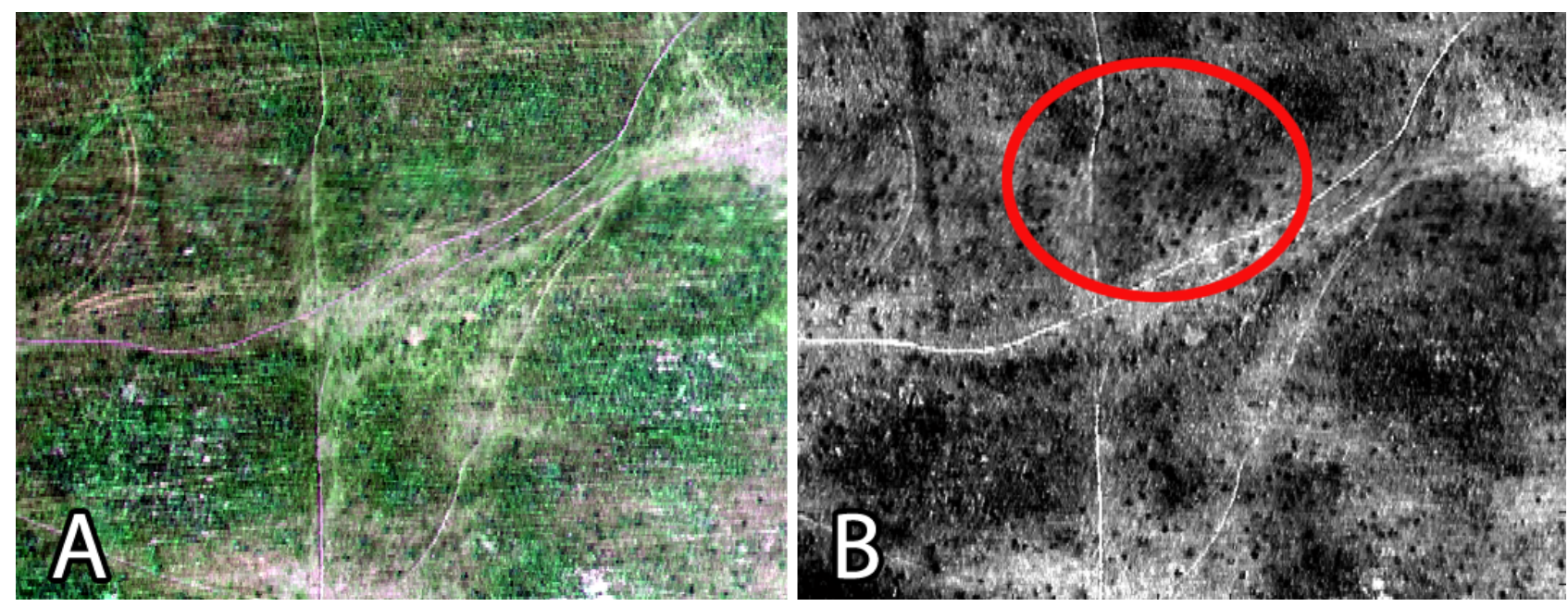

Figure 3: (A) Normal RGB image; (B) the same area visualised by the value of the shape parameter resulting from a gamma distribution fitting. Notice the darker vegetation spots not visible in version (A). Both images are histogram-stretched.

National Heritage Board (S), IBM VISTA-University of Birmingham (GB) and NIKU-Norwegian Institute for Cultural Heritage Research $(\mathrm{N})$.

\section{REFERENCES}

AtZBerger C, EILERs PHC. 2011. Evaluating the effectiveness of smoothing algorithms in the absence of ground refer- ence measurements. International Journal of Remote Sensing 32: 3689-3709.

Verhoeven G, Doneus M. 2011. Balancing on the Borderline - a Low-cost Approach to Visualize the Red-edge Shift for the Benefit of Aerial Archaeology. Archaeological Prospection 18: $267-278$. 